\title{
The SenseMS: Enriching the SMS Experience for Teens by Non-verbal Means
}

\author{
Alia K. Amin, Bram Kersten, Olga A. Kulyk, Elly Pelgrim, \\ Jimmy Wang, and Panos Markopoulos \\ Design School of User System Interaction, Eindhoven University of Technology, \\ Den Dolech 2, 5600 MB Eindhoven, The Netherlands \\ \{a.k.amin, b.t.a.kersten, o.a.kulyk, p.h.pelgrim, \\ c.m.wang \} atm. tue.nl \\ p.markopoulos@tue.nl
}

\begin{abstract}
The paper presents a design exploration into emotional communication through mobile phones for teenagers. A participatory design approach was followed, that lead to the development of two potential enhancements to text messaging services that are feasible with today's mobile phones. These enhancements refer to using MMS technology for: identifying callers through personalized avatars which are also coupled with context related information for the caller and using semi-automated text enhancements. Preliminary evaluation results are encouraging regarding the value of the emotional and contextual cues that can be conveyed in this way.
\end{abstract}

\section{Introduction}

Teeners use Short Messaging Service (SMS) to stay in touch with their peers and it is very popular among this group. The main reasons for this popularity are that it is fast, cheap, easy, and convenient. Teens consider the use of SMS as more convenient, because they can do it in private, silently in public places or late in bed. SMS also helps teens to avoid long and unwanted conversations and save costs.

However, short messaging has several drawbacks. The most obvious restriction is the absence of non-verbal communication support. In addition, text messaging lacks expressiveness [1] and human embodiment (e.g. usage of avatars in Instant Messaging [2]). In face-to-face situations, non-verbal aspects account for a great proportion of the total communication (e.g. facial expressions, body posture, gestures, etc.). Due to the fact that a receiver tends to interpret the intentions of the sender based on the nonverbal cues she/he perceives from the message, the absence of those makes the correct interpretation difficult for receivers, without sufficient indications.

In this paper we report the user study results and design concepts of a new messaging application for teens. Section 2 describes the user study of teens' behavior regarding informal communication. Section 3 presents our enhanced SMS design concept, the SenseMS. Section 4 describes the preliminary user evaluation, which focuses on comparing teens' understanding of emotion in using SMS and SenseMS. Section 5 concludes the paper and discusses perspectives in a view of recent developments. 


\section{User Study}

A user study was conducted to get insight into teens' communication and life style. Our aim was to gain insight into teens' communication needs, as well as to establish the necessary empathy with our target users. Two participatory design workshops were conducted, part of which was inspired by the Cultural Probes technique [3]. The probes were designed as creative tasks in a colorful booklet, which were adapted to teens' interests and preferences (see figure 1). Two groups of teens (one group of 4 girls and one group of 5 boys) participated in the workshops, which took place in their home environment.

The results showed that it is common for teens to add emoticons to their messages to express their emotions and intentions. Illustrative was that during the assignments a lot of pictures and cartoons of facial expressions were used to express how they were feeling. Still, it is essential to mention that, when they had a choice, the teens preferred to also include text. In addition, the workshops demonstrated that context information is an important aspect in communication for teens, because it was also noticed that often during phone calls (e.g. in the train or in shops) the teens first ask each other where they are.
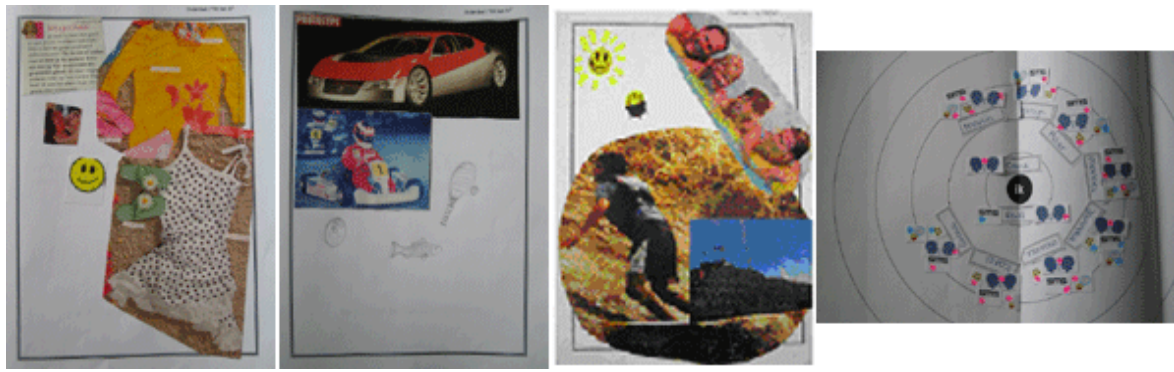

Fig. 1. The teen's craftwork from left to right: a result from "this is me" of girl (age 17) and of boy (age 15), a result from "the holiday" assignment, and a result of the "them and me" assignment

\section{The Design Concept}

From the findings of the user study and literature about teenagers' behavior, we propose an enhanced SMS design based on the following four elements, which are very essential in teen's communication:

1. Identity: The use of avatars is one way for people to visually represent themselves in the virtual world. Especially among teens, avatars are popular and widely used. What makes avatars favorable is that they give teens a possibility to be represented in a real or surreal (e.g. caricatures, cartoons, idols) way.

2. Personalization: When teens use Internet text applications (chat and instant messaging) they are very creative in expressing themselves with various text colors and sizes. Oksman et al. [4] found that teens have become large-scale consumers of personalized mobile services (logos and ringing tones). 
3. Facial expression: A crucial part of non-verbal communication is body language. Facial expressions especially help in the interpretation of the intention of the communication content.

4. Location awareness: The workshop showed that teens expressed the location information in the environment to elaborate their messages.

The complete design composes of two parts, the stamp and the enhanced text message. The stamp (see figure 2) is the first part the user sees when he/she receives a message. It provides the situational and environmental context (the stamp background) and the psychological context (the emotion displayed by the avatar). In addition, the sender's name, and the time and date the message was send are displayed as usual. This way the receiver of the message has a lot of information at a glance, before the actual text message is even read. Teens can personalize the avatar and the background picture. The avatar's facial expressions can be adjusted according to the user's preference.

The second part of the SenseMS is enhanced text. The sender of a SenseMS can customize the text appearance, by changing the background color, the font type, and the font color.

In addition to the above, the application filters the content of a text message as it is typed, prompting the user with suggestions for the presentation of special words. In a manner comparable to automatic word completion or detection of emoticons in instant messaging, words that are typically associated with a specific emotion will be provided with a pop-up providing different text styles and adorned with graphics. For example, like with the word "jarig" in figure 2.
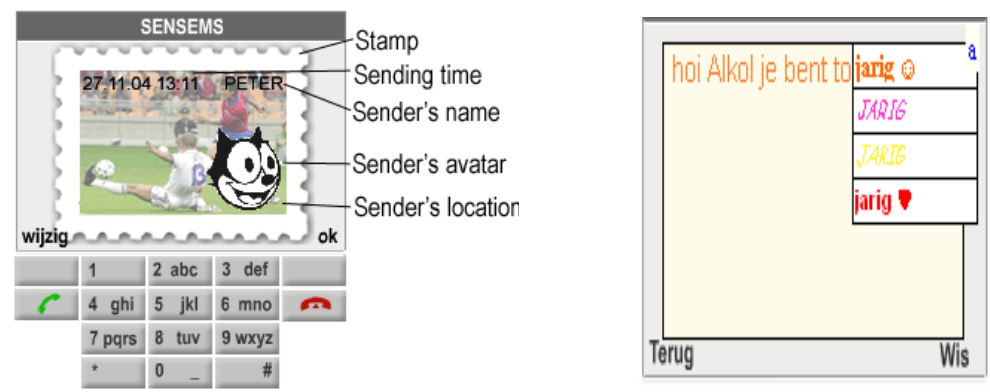

Fig. 2. The design of stamp (on the left) and enhanced text (on the right) of SenseMS application

\section{Prototype Evaluation}

Based on the design concept, we developed a SenseMS prototype on a Pocket PC platform to simulate the mobile phone usage. A preliminary evaluation was conducted with 8 teens to test if they could understand the emotion and context of the sender more easily using SenseMS compared to SMS. We asked participants to compose 
emotionally charged messages to each other and then interpret the emotion and context conveyed in the messages.

The test results show that teens interpreted the emotion conveyed in SenseMS better than SMS, especially negative emotions. Teens reported that they enjoyed receiving a SenseMS, because of the possibility to communicate emotions and context, which makes them feel closer. Teens also indicated different usage scenarios for SMS and SenseMS, namely sending ordinary messages with SMS and messages for special occasions with SenseMS, because the composing time for SMS is shorter than SenseMS.

\section{Conclusion and Future Work}

This study has identified shortcomings of current messaging services with regards to communicating emotions and contextual information regarding the caller. We have proposed two design concepts that can enrich non-verbal communication allowing the expression of emotion, the efficient communication of contextual cues and the personalisation of messages. Clearly, our evaluation was of a small scale and a more extensive field trial involving a fully functional service is needed to consolidate our findings. Further, an improved input method should be implemented in the application.

The concepts proposed were designed to be feasible with current technology. A direct improvement of today's services in line with our findings would be for network operators to provide real time context information that can be used to construct the Stamp as shown in this work. Future developments in context awareness through the use of sensor and GPS technology as, for example, explored by Marmasse [5], also promise to enrich the contextual information adding no extra tasks to the sender of the message.

\section{References}

1. Berg, S., Taylor, A.S., Harper, R.: Mobile Phones for the Next Generation: Device Designs for Teenagers. Proceedings of Human factors in computing systems, Ft. Lauderdale, Florida, USA (2002) 433 - 440

2. Takahashi, T., Bartneck, C., Katagiri, Y.: Show Me What You Mean - Expressive Media for Online Communities. Proceedings of the CHI Workshop on Subtle Expressivity of Characters and Robots, Fort Lauderdale (2003)

3. Gaver, B., Dunne, T., Pacenti, E.: Cultural probes. Interactions 1 (1999) 22-29

4. Oksman, V., Malinen, S., Utriainen, A., Rautiainen, P., Liikala, H.: Mobile Communication Culture of Children and Teenagers in Finland. A research project based on qualitative fieldwork 1997-2001, University of Tampere (2001)

5. Marmasse N., Schmandt S., Spectre D.: WatchMe: Communication and Awareness Between Members of a Closely-Knit Group. Proceedings of UbiComp, Nottingham, England (2004) 